\title{
Association Libre des Pathologistes Suisses
}

\section{2íe Assemblée annuelle à Neuchâtel, les 21-23 novembre 1958}

Freie Vereinigung der Schweizer Pathologen

2-i. Jahresversammlung in Neuenburg, 21.-23. November 1958 President

annueliJahrespråsident: $\mathrm{Ch}$. Hedinger

Liste des communications - Verzeichnis der Vorträge

Zollinger, H. U'.: Die hypertensive Arteriolopathie 262

Diskussion: Hedinger, Loustalot, Zollinger, Siebenmann, Cottier, Gloor Albertini, A. von: Zur

Morphologie der terminalen Strombahn im Granu-

lationsgewebe 285

Diskussion: Zollinger, von Albertini fiüttner, J. R.; Rondez, R. und Gassmann, R.:

Histochemische Unter-

suchungen an der toxisch geschíidigten Rattenleber 294

Diskussion: Hess, Scheidegger, Rüttner Zbinden,G.; Bächtold,H. P. und Studer, A.: Zur

Histopathologie experi-

menteller unspezifischer Entziindungen der Rattenpfote . . . 300

Diskussion: Rüttner, Studer, Hedinger Stünzi, H.: Zur Pathologie des Lungenkarzinoms bei

Hund und Katze 311

Diskussion: Sträuli, Slünzi, Berblinger, Hedinger Gloor, F. und Thölen, H.: Bilaterale

Nierenrindennekrosen (Spätstadium

bei einer Überlebenszeit von 92 Tagen) $\quad 318$

Diskussion: Zollinger, Siebenmann, Gloor, Cottier, Hedinger Hess, R. und Peurse, A. G. E.:

Renale Glueose-6-Phosphat-Dehydrogenase

und experimenteller Hochdruck der Ratte 329

Diskussion: Zollinger, Rüttner, Siebenmann, Hess (Schlußwort)

Riniker, P.: Experimentelle Knochenbildung 331

Diskussion: Rutishauser, Studer, Riniker (Schlußwort) Scheidegger, S.: Schwer rubrizierbare

Formen der Virusencephalomyelitis 337

Diskussion: Vetter, Scheidegger, Nicod Werthemann, A.: Über Spätschäden verschiedener

Organe durch Thoro-

trast und autoradiographischer Nachweis desselben .... 350

Diskussion: Zollinger, von Albertini, Rutishauser, Studer, Rüttner Aufdermaur, M. und

Brodhage, H.: Die experimentelle Rattenlepra . . 363

Dikussion: Berblinger, Aufdermaur Sträuli, P.: Was bedeuten die sogenannten Vogelaugen im Krebsgewebe? 368 Gardiol, D,: A propos du diagnostic différentiel histologique sur biopsies du foie entre ictère « medical » et ictère « chirurgical » . . . 375

Diskussion: Cottier, Gardiol, Gloor Cottier, H.; Gautier, E. und König, M. P.: Vermehrung und Polymorphic

azidophiler Zellen in der Adenohypophyse bei Gargoylismus . . 383 
Diskussion: Berblinger, Rutishauser, Cottier Kapanci, Y. et Veyrat, R.: Artériosclérose pulmonaire par thromboses

artérielles et artériolaires 392

Pathologie und Bakteriologie, Vol. 22, No. 3 (1959) 18 